\title{
COVID-19 and Beyond: Taming the Virtual Healthcare Tiger with Colored Petri Nets based Modeling and Simulation
}

\author{
Vijay Gehlot, Elliot B. Sloane \\ CS Department \& CEET, Villanova University \\ \{vijay.gehlot,elliot.sloane\}@villanova.edu \\ Michael Kirk, Eric Miller \\ Central Queensland Hospital and Health Service \\ \{michael.kirk,eric.miller\}@health.qld.gov.au
}

\begin{abstract}
The Coronavirus crisis has forced hospitals globally to develop new virtual service portals and systems to: 1) triage, diagnose, and manage new patients virtually for every clinical specialty at home as their symptoms emerge, avoiding COVID-19 exposure to patient or physicians, and hospitalization, as much as possible, and 2) discharge, track, and support recovered patients via homecare and virtual visits to free as many critical care beds as possible. This paper focuses on simulating and modeling an episode of care with innovative initial patient contact and triage processes using the Colored Petri Net (CPN) formalism to help optimize workflow, patient throughput, and overall system efficacy. The two patient triage programs under consideration are a health system in Australia and an orthopedic surgical program in the US. We describe our model for the US program. Our presented results establish a desired stratification of patients through a virtual musculoskeletal triage.
\end{abstract}

\section{Introduction}

Rapid growth of aging and chronic disease patients, and pervasive IoT and mHealth devices, caused hospitals to explore virtualization. Virtual Hospital pilots began long before COVID-19, but the pandemic radically accelerated innovation, adaptation, and adoption [1, 2, 3]. Pre-pandemic re-engineering sporadically affected well-established specialties like surgery, or small hospitals due to inertia and cost concerns, but COVID-19 forced most providers to suddenly implement virtualization transformations. However, significant concerns remain about patient outcomes, safety and satisfaction, clinical and fiscal efficacy, and operational and fiscal sustainability. The pandemic forced rapid ad hoc improvisation, but now
Dominic King, Jonathan Schaffer

Cleveland Clinic Foundation

$\{$ kingd2,schaffj\}@ccf.org

Nilmini Wickramasinghe

Epworth Health Care and Swinburne University nilmini.work@gmail.com

hospitals and physicians need robust re-engineered processes to ensure optimum, affordable, and efficient patient care.

The work reported in [4] identifies the role that contemporary computer modeling and simulation can play in the research and development to optimize emergency care delivery systems, noting "One underutilized approach to addressing problems in health care quality and value ... is through the use of computer simulation modeling." Also: "Computer simulation should be viewed as a necessary first step prior to implementation of a change in procedure or practice." Our presented work illustrates this process.

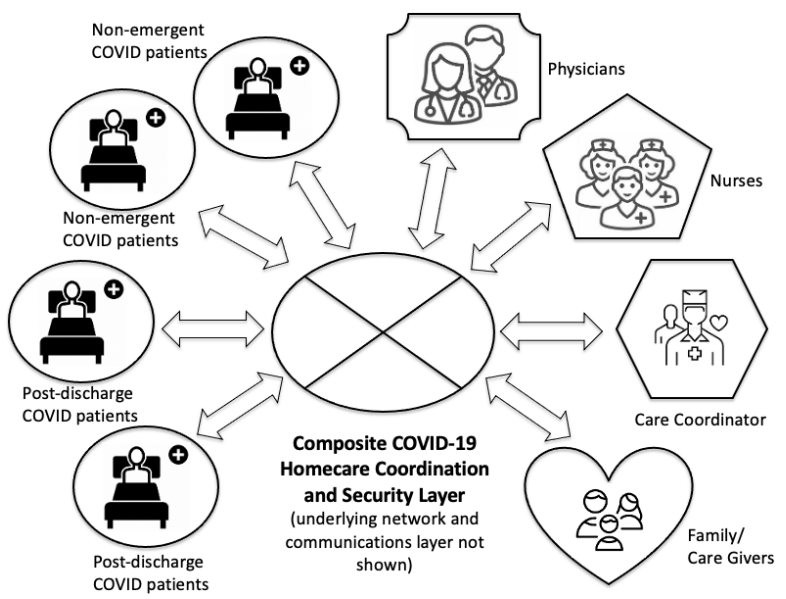

Figure 1. An illustrative COVID-19 Community Care Coordination Hub.

To understand and appreciate the complexities involved in a virtualized care, consider an illustrative care coordination hub shown in Figure 1. It shows COVID-19 patients being monitored and cared for remotely. For proper care, the physicians and nurses must rely on the coordination hub to keep them apprised of patient vital signs and complications. The hub must include family or other local/neighborhood 


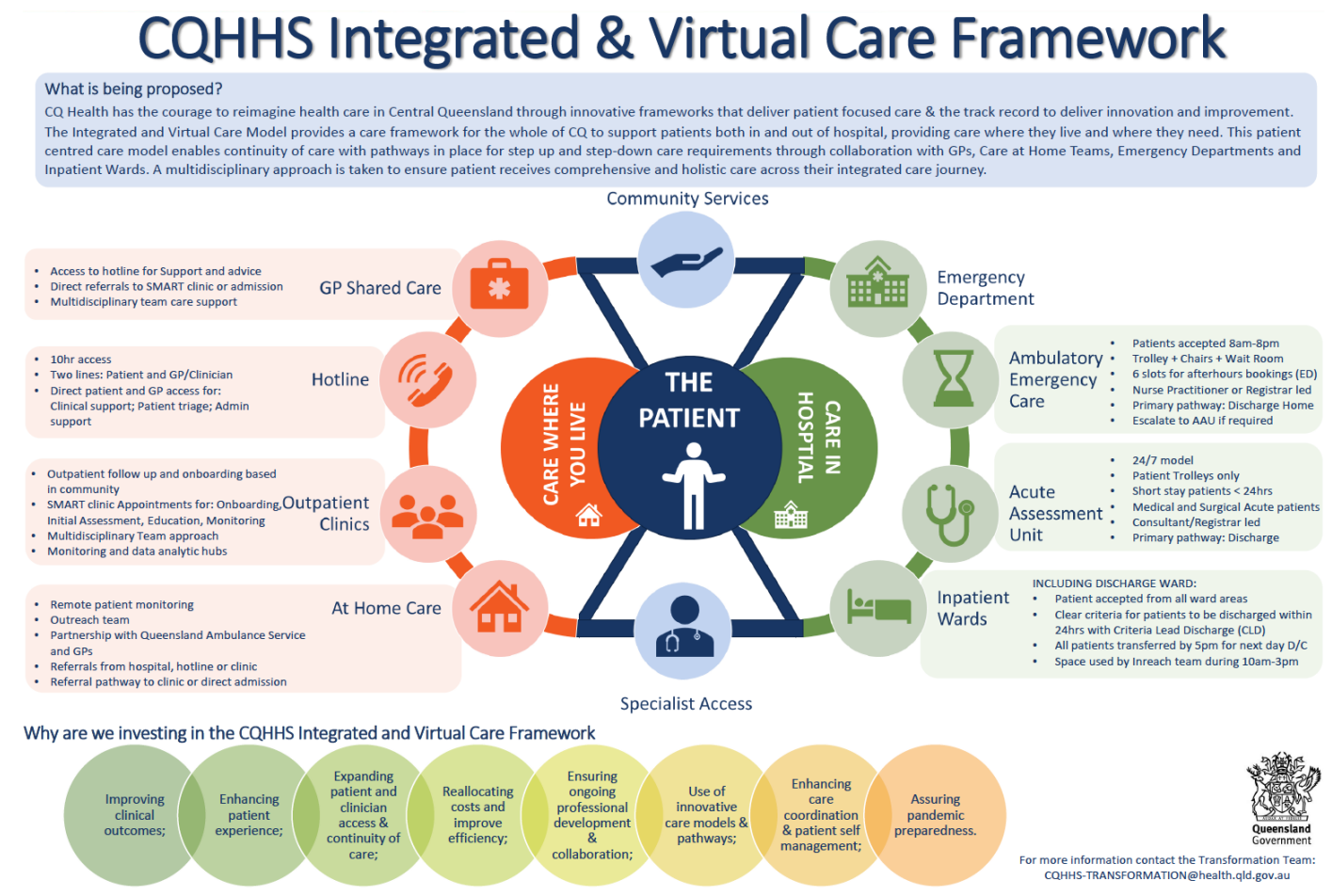

Figure 2. The Central Queensland Hospital and Health Service Integrated Virtual Care Framework (M. Kirk, personal communication, February 23, 2021).

caregivers, and may also include nursing homes, senior living homes, and, possibly even temporary COVID-patient field hospitals. The system must be able to handle complex patient care, including patients with urgent and emergent health crises that may require immediate physician intervention, hospitalization, emergency transport and care, surgery, radiology, or other hospital-based services. In essence, the present research addresses the question: "what if the patient is far too sick for home or community care?" The community care coordination team needs to be able to make a clear assessment that the patient's needs have exceeded their skills and services, and that the patient must be handed off to a triage program that is prepared and qualified to take the necessary steps to expedite emergency care.

Based on [5], we are following a case study approach with two exemplar cases-one in Australia and one in the US. Given the distinct healthcare systems in the two countries, by having two cases we also demonstrate the benefits of our approach for different healthcare systems. The first case is a hospital system in Queensland, Australia that needs to be ready to take charge of a sick patient and expedite the services provided, and the second case is a large orthopedic surgery practice that needs to determine if a serious auto or sports accident, or workplace injury, requires immediate intervention. In both cases, there are currently significant COVID-19 considerations, because infected patients may need to enter the hospital and system through a special pathway, and infection control considerations may also eliminate or limit any accompanying person with the patient.

For the Australian hospital, geographic distances between residences and hospital facilities make transportation and stabilization choices more difficult. A seriously ill or injured person may need to be moved to a smaller regional facility where an emergency physician can be guided by a specialist at a major trauma center. The trauma center thus becomes the hub, supporting local care via spokes. Patient transport may be done in stages, using ambulances, helicopters, or planes, as distance, weather, and resources allow. Many balancing trade-offs need to be considered, including the preference by many individualistic Australians living in spartan rural communities to stay at, or near their home, ranch, or family. Also, depending on the weather and terrain, subjecting the patient to transport to, and later, from, the hospital may be difficult, painful, expensive, or risky.

For the US-based orthopedic surgery program, there are additional unique considerations that the $\mathrm{CPN}$ model 
must address. The age of the patient (pediatric, adult, senior), prior orthopedic surgical history (e.g., major joint arthroplasty with a prosthetic joint infection or periprosthetic fracture), the existence of prior or simultaneous chronic or acute medical problems such as obesity, diabetes, or high blood pressure, the severity of pain, and physical risks such as falls. It might be inferred that the second case is an extension of the first case. That is, an Australian senior living independently on a ranch may fall off a horse, shattering a prior stable hip implant, so many of the US orthopedic surgical team's decisions may ultimately come into play in Australia. In the US, however, patients may access the specialists directly, depending on insurance and circumstances, whereas many Australians typically go via their general practitioner/primary care doctor to access surgeons.

Figure 2 shows the Australian virtual care coordination framework. The origin of this framework is the COVID-19 pandemic that necessitated unprecedented responses to patient care management to ensure they can provide continuity of care for patients regardless of their location while maintaining service business requirements. To ensure patients received the right care, at the right place, at the right time, contemporary clinical service models and modalities were explored and adopted by the healthcare provider. Although this virtual triage transformation was developed and implemented rapidly by necessity, it provides an opportunity to 'hold the gains' and use the lessons learned to adopt and embed these contemporary models into a sustainable service provision.

Figure 3 depicts a conceptual model that integrates virtual triage into patient access pathways of traditional orthopedic consultation, surgery, and emergency services, as reported in [6]. Again, the origin of this virtual triage model is the COVID-19 pandemic. Similar to the Australian case above, it presents opportunities for a long-term solution to providing healthcare services.

In both cases, several questions need to be answered before fully realizing the proposed changes. Overall the episode of care considerations can be evaluated through simulation prior to implementation. For example, will virtualization add value to the care of patients? What kind of patients? Will it be cost-effective? Will it be safe? What are the personnel requirements? What are the system requirements? Should online services be deployed in the cloud or invest in servers?

As mentioned in [4] above, a robust modeling and simulation approach that goes beyond the conceptual models of virtual care can help answer such questions. In this paper, we give details of constructing a Colored Petri Nets (CPNs) based simulation model of the conceptual diagram shown in Figure 3 and described in [6].

The remainder of this paper is organized as follows. In Section 2, we review some related work in the area of healthcare modeling and simulation including some work in the context of the COVID-19 pandemic. In Section 3, we give an overview of CPN and introduce the vocabulary of the $\mathrm{CPN}$ modeling language utilizing a simple example. Owing to page limits, this paper in Section 4 focuses on a hierarchical CPN model of the surgical virtual care shown in Figure 3. Note that the surgical workflow is the "Specialist Access" shown in the virtual care framework of Figure 2. Section 5 provides details of our simulation run and results. Finally, in Section 6, we present our conclusions and describe the future work.

\section{Related Work}

Early work on engineering medical processes and improving safety through the use of process modeling and analysis is reported in [7] and [8]. Other early works identified uses of Colored Petri Net models for intelligent wireless medical device networks for flexible hospital capacity management $[9,10]$. Related work reported in [11] highlights the value of process modeling in healthcare.

Work reported in [12] evaluates orthopedic department of a Belgian hospital focusing on the impact of outages on the effective utilization of resources and the flow-time of patients. A simulation study to increase the throughput at an endoscopy center is described in [13]. A non-hierarchical CPN model dealing with patients' workflow in heart clinics is presented in [14].

A recent work reported in [15] provides details of a stochastic web-based decision support tool for hospital resource allocation during the COVID-19 pandemic. Another recent work on using community care coordination networks to minimize hospitalization of COVID-19 patients is presented in [16].

\section{Colored Petri Nets Primer}

In this section, we briefly describe the basic elements of Colored Petri Nets (CPNs) through a simple example. Readers interested in details of the CPN formalism, including formal definitions and theoretical foundations, may refer to [17], [18], and [19]. A condensed introduction to CPNs is available in [20]. Two examples-based tutorial introductions to CPN and underlying details are provided in [21] and [22].

At the very basic level, a Colored Petri Net consists of places (depicted as circles or ovals), transitions 


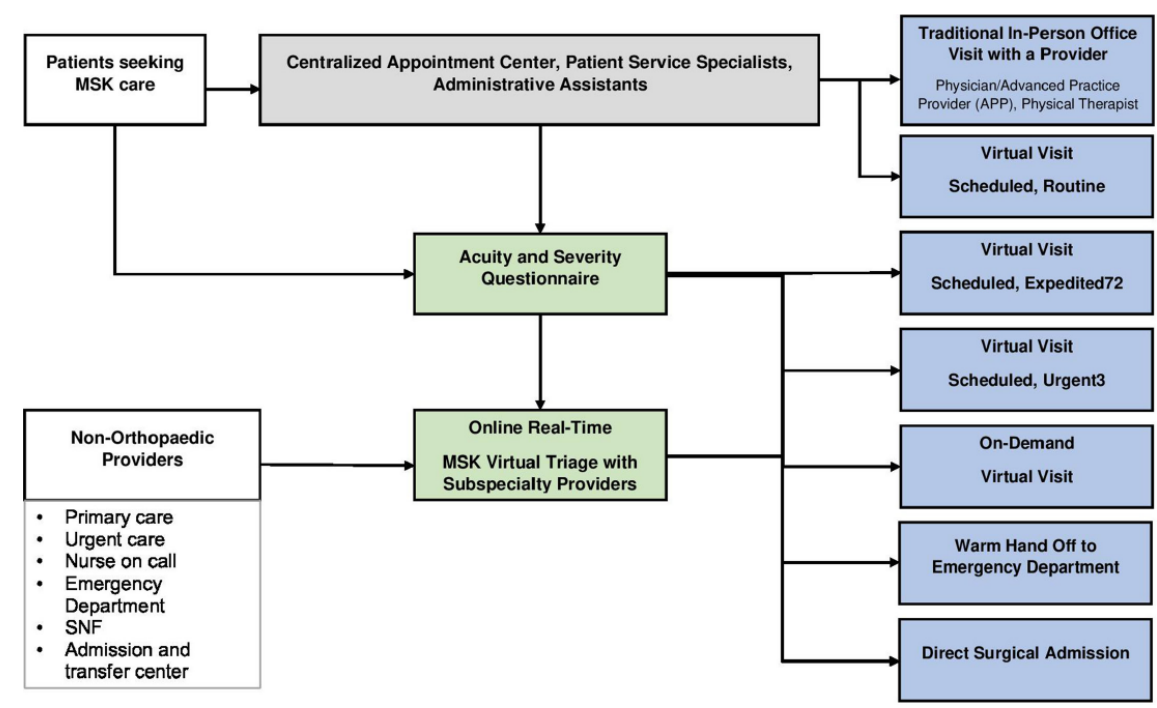

Figure 3. Integration of the virtual triage into traditional patient access pathways as given in [6].

(depicted as rectangles or bars), and arcs (depicted as arrows) that connect a place to a transition or a transition to a place [23]. The only syntactic restriction is that two places cannot be directly connected without an intervening transition and two transitions cannot be connected directly without an intervening place. A net can have any number of places, transitions, and arcs. It is not required that the entire net be one single connected graph. Figure 4 shows a very basic net consisting of two places (P1 and P2) and one transition (T1). The interpretation of these net elements depends on the system being modeled. For example, we can interpret P1 as "Printer Available", T1 as "Print Job Arrives", and P2 as "Printer Busy". However, the same net may be given a different interpretation with P1 as "Healthy", T1 as "Bug Bites", and P2 as "Sick". Therefore, a net gives us an abstraction mechanism to see similarities among seemingly different systems. Thus, from a design and analysis perspective, it is possible to transfer interesting properties and results from one system to another.

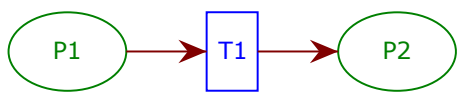

Figure 4. A basic CPN with two places and one transition.

There are two required attributes associated with a place-its name and type (called colorset in CPN parlance). Places may have tokens, which are values of the associated colorset. The default colorset for a place is UNIT, which is a type containing exactly one value and denoted by (). In fact, as a container, a place is a multi-set allowing multiple copies of the same value. For example, in describing a printing system, we may have a scenario with two laser printers and three ink-jet printers. In the CPN notation, the multi-set $\{a, b, a, c, c, a\}$ is written $3^{\prime} \mathrm{a}++1{ }^{\prime} \mathrm{b}++2^{\prime} \mathrm{C}$ denoting six tokens.

A place with a token is termed marked and describes a local state of the system. The distribution of tokens across places in a given net is called a marking and describes the collective global state of a system. The dynamics (semantics) of a CPN is defined by the firing rule where the firing of a transition removes tokens from its input place and adds tokens to its output place. The tokens removed and tokens added are governed by the expressions on the input arcs and output arcs of a transition, respectively. For the default case of UNIT, omitting arc inscription would default to $I^{\prime}()$ and a positive integer $n$ would evaluate to $n$ ' ( ) . The simplest of these expressions is just a constant or a variable. In the latter case, the variable will be bound to one of the values (tokens) residing in the input place. In general, arcs may be inscribed by more complex expressions that evaluate to a multi-set representing tokens to be removed or added. A transition is termed enabled if all of its input expressions can be evaluated to a multi-set based on the available tokens in the associated places. Note that per the semantics described above, only an enabled transition may fire. The firing of a transition is an abstraction of the occurrence of an event and the movement of tokens describes state changes. This small basic vocabulary and simple semantics render CPNs very flexible in terms of application domains for modeling systems of varied nature. 


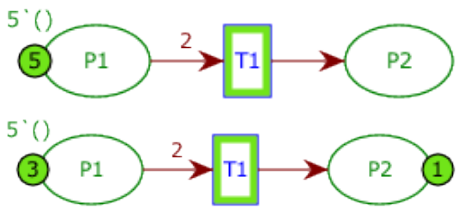

Figure 5. An example CPN before and after the firing of transition $\mathrm{T} 1$.

The only required inscription of a transition is its name. An optional inscription is a guard (Boolean condition) that can be used for finer control of transition firing. We will see an example later on in this paper. An optional attribute of a place is its initial marking, which is written as a multi-set. Figure 5 shows a net with 5 tokens in place P1 as determined by its initial marking 5 ' ( ). When the transition T1 fires, it removes 2 tokens from place $\mathrm{P} 1$ and deposits 1 token in place $\mathrm{P} 2$, as determined by the associated outgoing and incoming arc inscriptions, respectively. Note the new token count of 3 in $\mathrm{P} 1$ and 1 in $\mathrm{P} 2$ as the result of $\mathrm{T} 1$ firing. Under a different interpretation, the given CPN captures a basic step (or requirement) of a vending machine whereby one needs to supply 2 quarters for it to dispense 1 candy bar.

CPNs support a mechanism of modules for hierarchically constructing models of large systems. The hierarchy and module concept of CPNs allow different levels of abstraction that are inherent in most systems. The graphical representation makes it easy to see the basic structure of a complex CPN model, that is, understand how the individual subsystems interact with each other. The creation of hierarchical nets is based on the simple idea that any transition can be replaced or substituted by a (sub) net that details the activities underlying it. Such transitions are called substitution transitions in the CPN parlance. Pictorially, a substitution transition is drawn with double rectangles. The top-down approach starts with a non-hierarchical net containing transitions as high-level modules and their interconnections that capture the architecture of the system in terms of its subsystems and their interfaces. This allows the building of a hierarchical model incrementally and makes the approach very practical. Furthermore, not all transitions of interest need to be expanded to simulate and analyze a CPN model.

CPN models can be made with or without explicit reference to time. Untimed CPN models are usually used to validate the functional/logical correctness of a system, while timed CPN models are used to evaluate the performance of the system. CPN models are built using CPN Tools which is a graphical software tool for creating, editing, simulating, and analyzing models. The CPN Tools software can be downloaded from http: //cpntools.org. It also provides detailed online documentation and installation options.

\section{CPN Model Details}

For this project, we constructed a hierarchical executable CPN model of the conceptual virtual triaging of patients shown in Figure 3. To convert it to a CPN, we create a transition for each of the blocks and connect those with intervening places. The resulting top-level net of the hierarchical model is shown in Figure 6. The tokens that flow in this net represent patients. To be able to encode all patient-related processing we define a set of attributes based on the details given in [6]. A patient is then identified by a unique ID number and her age together with her clinically relevant attributes. The complete set of associated color set declarations are given below:

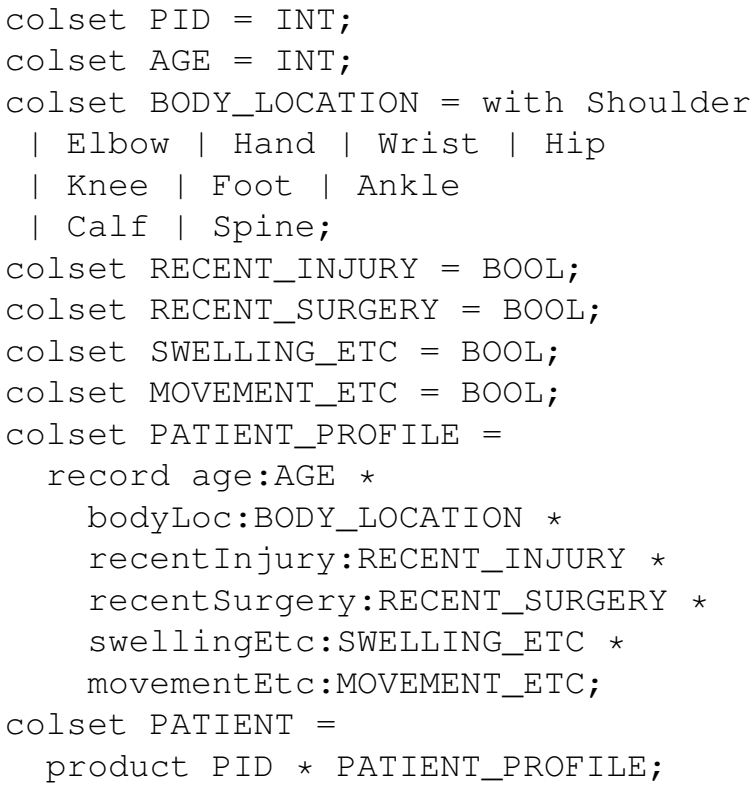

Withe these declations, the token $(3$, age $=45$, bodyLoc $=$ Hip, recentInjury = true, recentSurgery $=$ false, swellingEtc $=$ true, movementEtc $=$ true $\}$ ) represents a patient aged 45 with ID number 3 who has had a recent injury to her hip. There is swelling of the injury area but the patient has not had a recent surgery of the hip and can move. The overall patient flow and triaging described in [6] is as follows:

"Patients seeking musculoskeletal care are introduced by contacting the centralized appointment desk originating most from a provider referral, emergency department (ED), or urgent care consults. Patients are diverted to a virtual short questionnaire that can be administered electronically or 


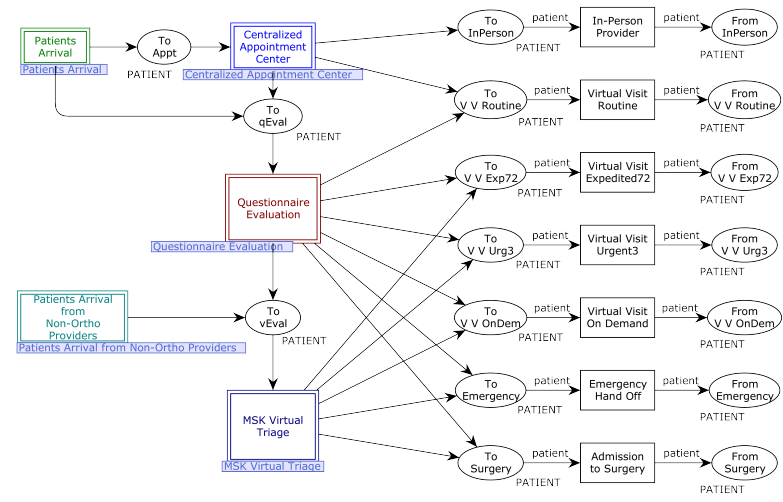

Figure 6. The top-level module of the hierarchical CPN model capturing the flow that is consistent with Figure 3. An exploded view with details is shown in Figure 12.

via telephone. The questionnaire would assess the acuity, severity or urgency and a secondary questionnaire that includes basic patient demographics, pertinent comorbidities, and key elements would provide insight into the nature of the patients' complaints, including type, location, character, acuity, recent surgical history, and newly obtained imaging. An acuity and urgency-based stratification of patients would be conducted based on outcomes of the virtual musculoskeletal triage questionnaire, where patients with chronic conditions and established follow-up patients are offered routine virtual visits according to availability and geographical location. Conversely, derangements that are deemed to be acute, especially among patients with recent surgical history, are directed to a virtual musculoskeletal triage channel where a live interview with a musculoskeletal provider would guide down-stream disposition. Based on the results of the triaging questionnaire, coupled with providers assessment, patients would be referred to an urgency-appropriate disposition ranging from direct surgical admission, referral to the emergency department (ED) or orthopaedic acute care centre after direct surgical admission, referral to the emergency department (ED) or orthopaedic acute care centre after direct notification, and provision of a plan-ofcare, or requesting appropriate imaging.
Alternatively, patients could be scheduled for an on-demand (immediate), urgent (same-day), expedited (within 72 hours), or routine virtual visit with the appropriate member of an orthopaedic clinical care team."

We use this description to create sub-modules for the substitution transitions Centralized Appointment Center, Questionnaire Evaluation, and MSK Virtual Triage shown in Figure 6. We have two additional substitution transitions, namely, Patients Arrival and Patients Arrival from Non-Ortho Providers. These are used to generate tokens representing patients for simulation purposes.

Figure 7 shows the sub-module that corresponds to the substitution transition Patients Arrival. Note that, per Figure 3 and associated description, if a patient arrives after hours then she bypasses the centralized appointment facility and is directly presented with the online questionnaire. The place NextID in Figure 7 is used to assign a unique ID. Timed tokens are used for patient arrival and patients arrive with an exponential delay for present purposes. If an actual log of patient arrivals is available, then the input/output facility of the CPN Tools software can be used to drive the arrivals based on that log. The transition Next Patient BH fires if the associated guard businessHours() is true. In this case, the patient token is placed in the To Appt outgoing place. Otherwise, the guard afterHours() evaluates to true and places the token in To qEval place for direct questionnaire-based evaluation and bypassing the centralized facility. The sub-module associated with Patients Arrival from Non-Ortho Providers is similar except it always sends the patient to To $v E v a l$ for virtual triage which is available $24 / 7$.

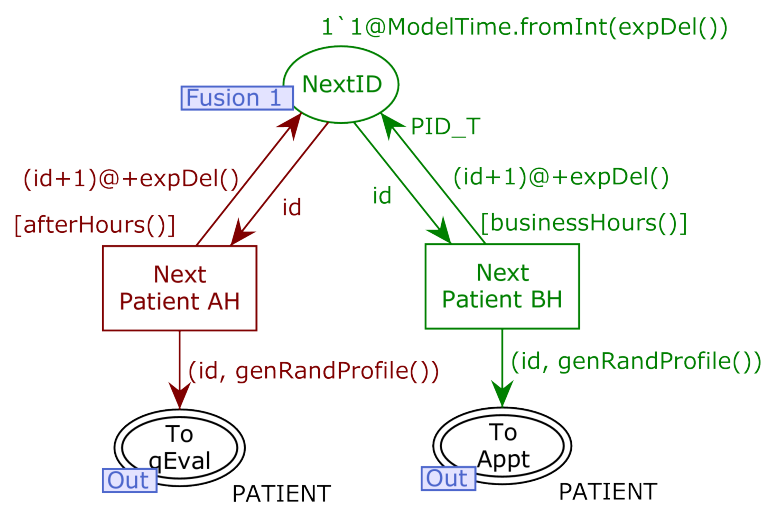

Figure 7. The Patients Arrival sub-module for generating tokens representing patients with different profiles.

Figure 8 shows details of the sub-module associated 
with the Centralized Appointment Center substitution transition. For present purposes, we are only focusing on virtual triage, hence patients are not diverted to an in-person consultation. This is indicated by the arc inscription empty. For a routine virtual visit, the decision is made at the end of the questionnaire. The place Available Staff is used to account for human resources. Model parameters can be changed to account for desired allocation and availability of staff. Once a patient is registered, she is then directed to the online questionnaire for initial evaluation and triage, which is achieved by the firing of the Direct Patient transition.

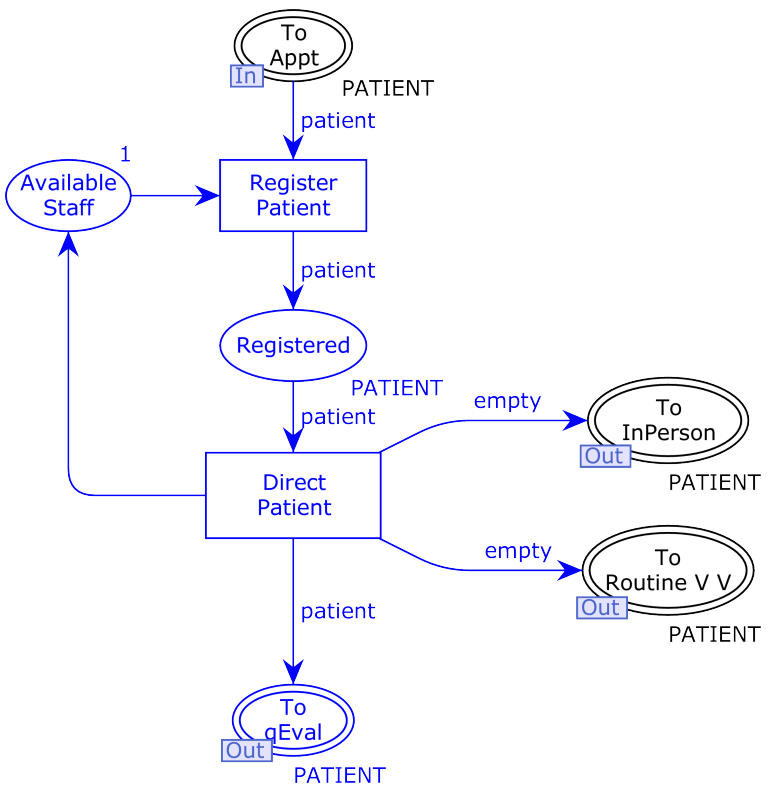

Figure 8. The Centralized Appointment Center sub-module for initial evaluation.

Figure 9 shows details of the sub-module associated with the Questionnaire Evaluation substitution transition. The place System Availability is used to account for system resources. This will allow us to determine system overload situations as well as usage costs for cloud-based services. Model parameters can be used to fine-tune these resources based on patient traffic. Once a patient has done answering the questions, the system evaluates and stratifies the patient based on her profile and protocols in place. For example, if the patient is calling about the elbow and has not had a recent injury or surgery then she is diverted to the routine virtual visit. These details are encoded in the function $q E v a l()$. It returns a boolean vector with exactly one value being true reflecting the outcome of the evaluation. This function gets called when the transition Evaluate and Stratify fires. Patients needing further evaluation are sent for a virtual triage by adding a token to the place To $v E v a l$ as a result of firing this transition.

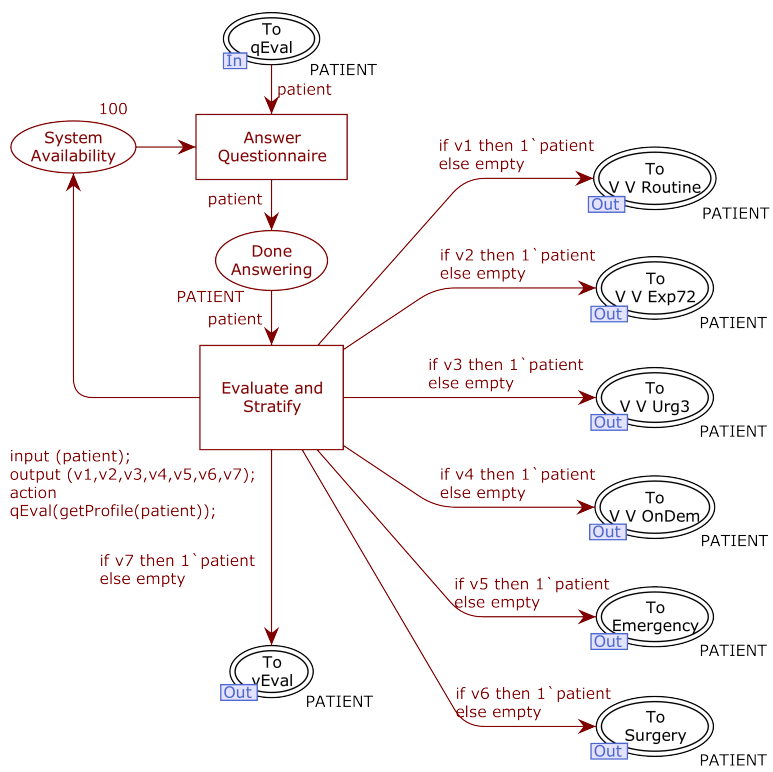

Figure 9. The Questionnaire Evaluation sub-module for the initial stratification of patients based on their profile.

Figure 10 shows details of the sub-module associated with the MSK Virtual Triage substitution transition. Since virtual triage requires the availability of both the system as well as trained staff, we account for these resources separately via places System Availability and Online Staff Availability. Both resources remain occupied until the end of triaging. Patients are triaged based on their profile and applicable protocols. These details are encoded in the function $v E v a l()$ which gets evaluated when the transition Do Virtual Triage fires. Coding these evaluations as separate functions makes it possible to implement and test different protocols without altering the underlying model.

Since [6] does not contain the details of the block of activities associated with the stratification, we have not expanded those transitions into underlying sub-modules. As mentioned before, we can still simulate and analyze the net built so far. We provide sample runs and results of the presented model next.

\section{Results}

The CPN Tools software provides a built-in facility to generate a simulation report which records every transition and bindings during a simulation run. The CPN Tools software also provides customizable monitoring facilities to extract relevant data of interest during a simulation run since monitors can be associated 


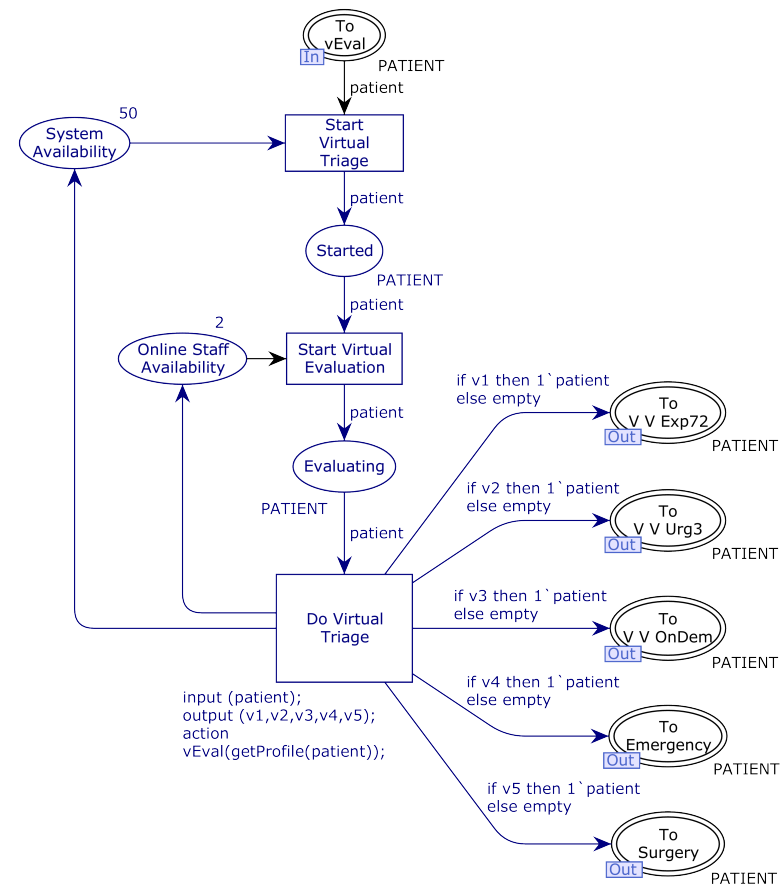

Figure 10. The MSK Virtual Triage sub-module for the final stratification of patients based on their profile.

with any subnet of interest [24]. For example, we can track patients with a specific profile using monitors. For present purposes, we used the simulation report since we were only interested in the overall stratification behavior of the model.

Figure 11 shows a portion of the simulation report that is auto-generated by the CPN Tools software during a simulation run of the presented model. At step 40, a patient with ID 10 directly enters the virtual triage. At step 41, another patient with ID 11 enters the system but this time through the centralized facility. However, since the model time evaluated to after-hours, patient with ID 11 is sent directly to answer the online questionnaire at step 42. Moving forward, we see that patient with ID 10 starts the virtual triage at step 45 . At step 46, a patient with ID 11 is evaluated. The report shows the values of variables $v 1$ through $v 7$. Only $v 7$ has the value true. Thus the inscription if $v 7$ then 1 'patient else empty on the outgoing arc from the transition Evaluate and Stratify to the place To vEval will be the patient token and it will get deposited in the output place To vEval. In effect, this implies that the patient is sent for the virtual triage since her profile indicates that she has had a recent surgery as well as a recent injury and has swelling and is unable to move.

Figure 12 shows the model state at the end

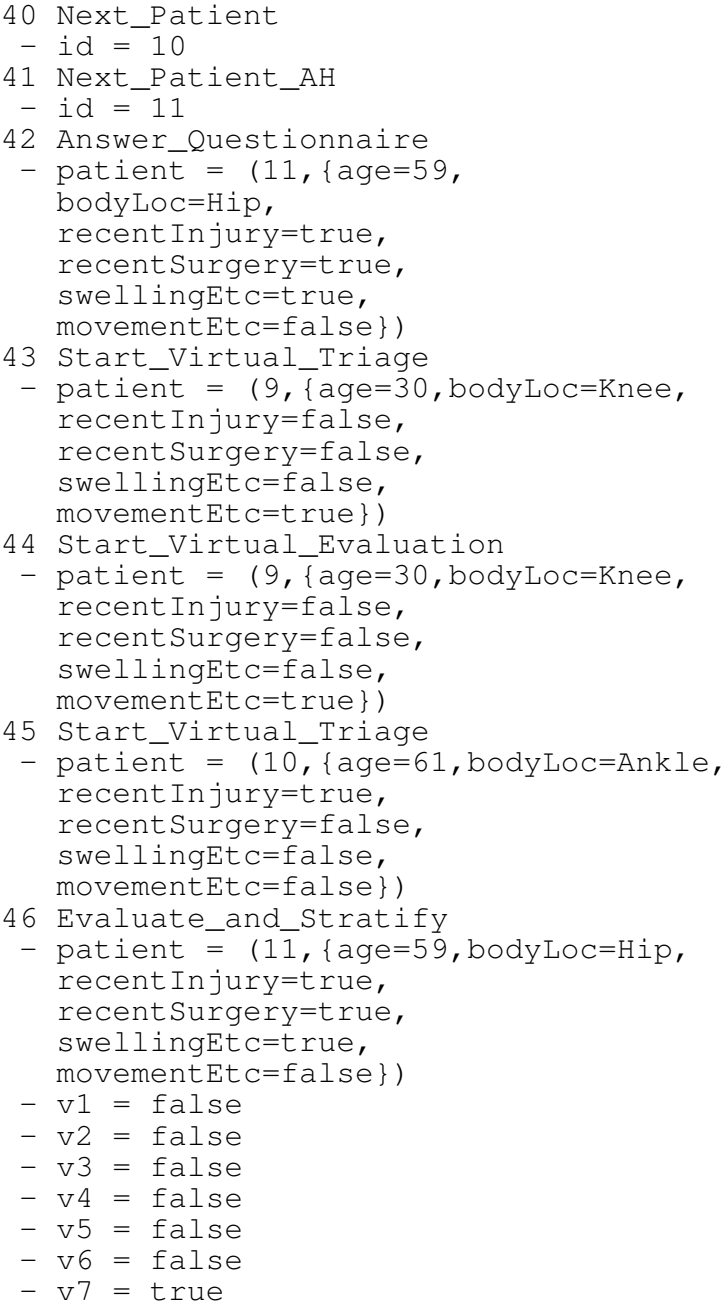

Figure 11. A portion of the simulation report auto-generated during a simulation run showing patient flow.

of a simulation run showing patient stratification through virtual triage. In this snapshot we see that the token $(20$, age $=39$, bodyLoc $=$ Foot, recentInjury = false, recentSurgery = false, swellingttc = true, movementetc = false) ends up in routine virtual visit because, although the patient has swelling and lacks movement, there is no recent injury or surgery. On the other hand, the token $(2$, age $=24$, bodyLoc $=$ Shoulder, recentInjury $=$ true, recentSurgery = false, swellingEtc = true, movementetc = false) ends up in urgent care. This is because the associated patient has a recent injury, has swelling, and is unable to move her shoulder. 


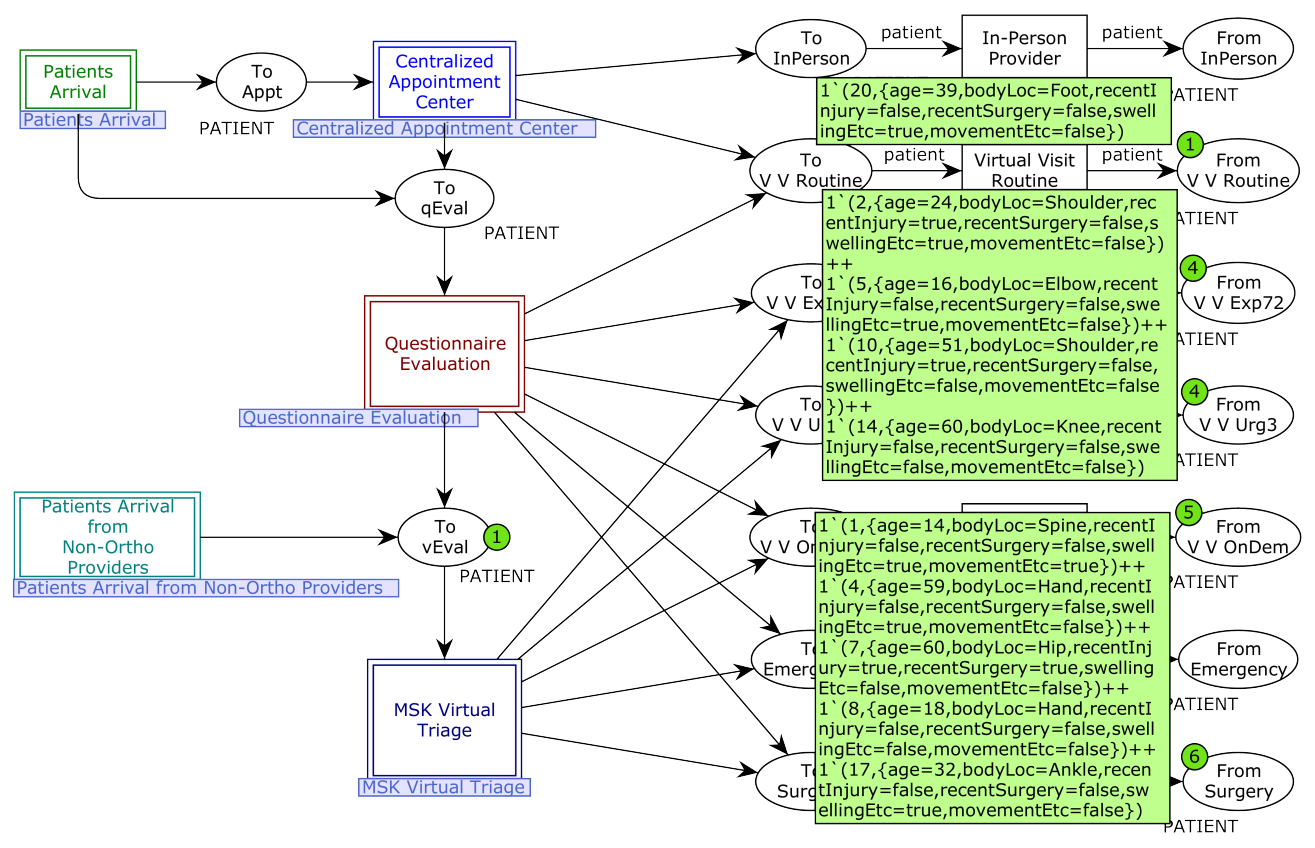

Figure 12. CPN model state at the end of a simulation run showing patient stratification through virtual triage.

\section{Conclusion and Future Work}

The COVID-19 pandemic forced hospitals around the globe to develop new virtual customer service portals and systems for two purposes: 1) to triage, diagnose and manage new medical patients for virtually every clinical specialty at home as their symptoms emerge, avoiding COVID-19 exposure to patient or physicians, and hospitalization, as much as possible, and 2) to discharge, track, and support recovered patients via homecare and virtual visits to free as many critical care beds as possible for incoming patients without impairing patient recovery. We discussed details of two virtual patient care and triage conceptual models that arose as a result of the COVID-19 pandemic. However, as noted in the paper, a conceptual model cannot help answer questions related to performance, patient safety, cost-effectiveness, resource constraints, etc. We presented details of how to build and simulate a Colored Petri Nets (CPNs) based hierarchical executable model. Utilizing the rich vocabulary of the CPN formalism, we were able to define informative tokens that capture full details of a patient's profile for musculoskeletal virtual care. These details are crucial to the verification and validation of a simulation model. We presented details of our simulation that showed the desired stratification of patients with differing profiles.

Our future work includes extending the presented model with remaining care workflows and instrumenting it with monitoring facilities for detailed data collection and analysis. We are also working on getting access to relevant hospital data for model verification and validation. Utilizing the external process communication capability of the CPN Tools software, we also want to add a 3-D visualization layer that could be used for training purposes. In the longer term, we want to explore multiple opportunities to implement a virtual visit in place of a traditional in-person office visit across the entire orthopedic episode of care. In parallel, we want to modify the presented model to capture critical care pathways of the conceptual model shown in Figure 2.

The orthopedic practice is using the CPN model to assess prospectively the impact, costs, and constraints if they vary triage resources based on time of day, holiday seasons, and other variable. That can allow them to optimize staff and information system resources in ways that ensure adequate patient processing flows without excessive expense. In the era of value-based medical care decisions by patients and payers, and fixed, bundle-payment restrictions for a growing number of orthopedic surgeries like hip and knee arthroplasties, the orthopedic practice needs to assure high patient and payer satisfaction while optimizing operational costs [6].

Lastly, Figure 13 shows a proposed triaging solution by the Central Queensland Hospital and Health Service (CQHHS). Although the overall flow is different from the model discussed here, the basic characteristics remain the same. In particular, some of the sub-modules 


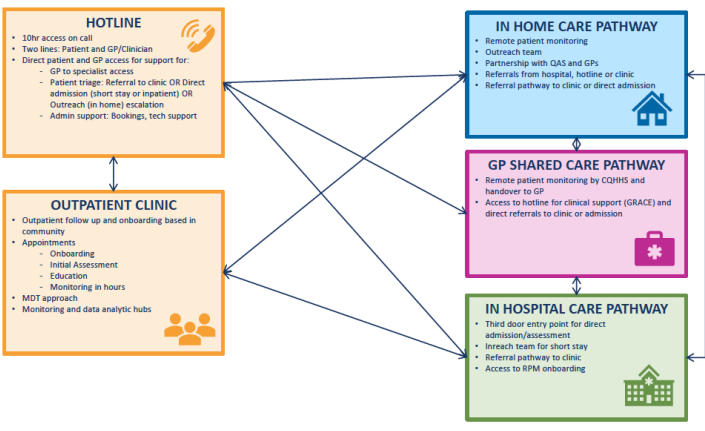

Figure 13. A proposed triage model by the Central Queensland Hospital and Health Service.

discussed here can be easily modified and reused. Furthermore, our model declarations for representing patients can be extended to accommodate different patient profiles including non-clinical attributes such as location.

\section{References}

[1] W. R. Hersh and R. E. Hoyt, Health Informatics: Practical Guide, Chapters 13 and 17. Lulu Publications, 7th ed., 2018.

[2] US CMS, "Acute hospital care at home, 2020." https://qualitynet.cms.gov/acute-hospital-care-at-home.

[3] UK NHS, "Covid virtual wards, 2021." www.england.nhs.uk/nhs-at-home/covid-virtual-wards/.

[4] L. F. Laker, E. Torabi, D. J. France, C. M. Froehle, E. J. Goldlust, N. R. Hoot, P. Kasaie, M. S Lyons, L. H. BargWalkow, M. J. Ward, and R. L. Wears, "Understanding emergency care delivery through computer simulation modeling," Acad Emerg Med., vol. 25, no. 2, pp. 116-127, 2018 .

[5] R. K. Yin, Case Study Research: Design and Methods. Thousand Oaks, CA: SAGE Publications, 5th ed., 2014.

[6] D. King, A. K. Emara, M. K. Ng, P. J. Evans, K. Estes, K. P. Spindler, T. Mroz, B. M. Patterson, V. E. Krebs, S. Pinney, N. S. Piuzzi, and J. L. Schaffer, "Transformation from a traditional model to a virtual model of care in orthopaedic surgery: COVID-19 experience and beyond," Bone \& Joint Open, vol. 1, no. 6, pp. 272-280, 2020.

[7] S. Christov, B. Chen, G. S. Avrunin, L. A. Clarke, L. J. Osterweil, D. Brown, L. Cassells, and W. Mertens, "Rigorously defining and analyzing medical processes: An experience report," in Models in Software Engineering (H. Giese, ed.), (Berlin, Heidelberg), pp. 118-131, Springer Berlin Heidelberg, 2008.

[8] L. J. Osterweil, G. S. Avrunin, B. Chen, L. A. Clarke, R. Cobleigh, E. A. Henneman, and P. L. Henneman, "Engineering medical processes to improve their safety," in Situational Method Engineering: Fundamentals and Experiences (J. Ralyté, S. Brinkkemper, and B. Henderson-Sellers, eds.), (Boston, MA), pp. 267-282, Springer US, 2007.

[9] V. Gehlot and E. B. Sloane, "Ensuring patient safety in wireless medical device networks," Computer, vol. 39 , no. 4, pp. 54-60, 2006.
[10] E. B. Sloane and V. Gehlot, "Use of coloured Petri net models in planning, design, and simulation of intelligent wireless medical device networks for safe and flexible hospital capacity management," International Journal of Networking and Virtual Organisations, vol. 4, no. 2, pp. 118-129, 2007.

[11] T. Eldabi, "Implementation issues of modeling healthcare problems: Misconceptions and lessons," in Proceedings of the 2009 Winter Simulation Conference (WSC), pp. 1831-1839, 2009.

[12] S. Creemers and M. R. Lambrecht, "Modeling a healthcare system as a queueing network: The case of a belgian hospital," tech. rep., Department of Decision Sciences and Information Management, Katholieke Universiteit Leuven, Belgium, 2007.

[13] M. A. Centeno, H. R. Dodd, M. Aranda, and Y. Sanchez, "A simulation study to increase throughput in an endoscopy center," in Proceedings of the 2010 Winter Simulation Conference, pp. 2462-2473, 2010.

[14] M. Zeinalnezhad, A. Chofreh, F. Goni, J. Klemeš, and E. Sari, "Simulation and improvement of patients" workflow in heart clinics during covid-19 pandemic using timed coloured petri nets," International Journal of Environmental Research and Public Health, vol. 17, no. 22, pp. 1-18, 2020.

[15] S. Brüggemann, T. Chan, G. Wardi, J. Mandel, J. Fontanesi, and R. R. Bitmead, "Decision support tool for hospital resource allocation during the covid-19 pandemic," Informatics in Medicine Unlocked, vol. 24, pp. 1-7, 2021 .

[16] E. B. Sloane, V. Gehlot, N. Wickramasinghe, and R. Silva, "Using community care coordination networks to minimize hospitalization of covid-19 patients," in Proceedings of the SoutheastCon 2021, pp. 1-4, 2021.

[17] K. Jensen, "Coloured Petri nets and the invariant method," Theoretical Computer Science, vol. 14, no. 3, pp. 317-336, 1981.

[18] K. Jensen, "An introduction to the theoretical aspects of coloured Petri nets," in A Decade of Concurrency (J. W. de Bakker, W.-P. de Roever, and G. Rozenberg, eds.), vol. 803 of Lecture Notes in Computer Science, pp. 230-272, Berlin-Heidelberg: Springer-Verlag, 1994.

[19] K. Jensen and L. M. Kristensen, Coloured Petri Nets. Modelling and Validation of Concurrent Systems. Berlin-Heidelberg: Springer-Verlag, 2009.

[20] K. Jensen and L. M. Kristensen, "Colored Petri nets: A graphical language for formal modeling and validation of concurrent systems," Communications of the ACM, vol. 58, no. 6, pp. 61-70, 2015.

[21] V. Gehlot and C. Nigro, "An introduction to systems modeling and simulation with colored Petri nets," in Proceedings of the 2009 Winter Simulation Conference, pp. 104-118, 2010.

[22] V. Gehlot, "From Petri nets to colored Petri nets: A tutorial introduction to nets based formalism for modeling and simulation," in 2019 Winter Simulation Conference (WSC), pp. 1519-1533, 2019.

[23] W. Reisig, Understanding Petri Nets. Berlin-Heidelberg: Springer-Verlag, 2013.

[24] B. Lindstrøm and L. Wells, "Towards a monitoring framework for discrete-event system simulations," in Proceedings of the Sixth International Workshop on Discrete Event Systems (WODES'02), pp. 127-134, IEEE Computer Society, Institute of Electrical and Electronics Engineers, Inc., 2002. 